\title{
INTERCULTURALIDAD EN SALUD: COMPETENCIAS EN PRÁCTICAS DE SALUD CON POBLACIÓN INDÍGENA ${ }^{1}$
}

\section{INTERCULTURALITY IN HEALTH: COMPETENCE IN HEALTH PRACTICES WITH INDIGENOUS POPULATION}

\author{
Felipe Nayip Hasen NarvÁez*
}

\begin{abstract}
RESUMEN
Este artículo pretende discutir y reflexionar en torno a la manera en la cual los servicios del sistema de salud inmersos en territorios con alta presencia de comunidades mapuche, se adecuan a la idiosincrasia cultural de esta población, abordando cuáles son las dificultades con que los servicios de salud se encuentran a la hora de ejercer la práctica de atención de salud en contextos pluriétnicos, en donde la salud es entendida de forma holística, llevando necesariamente a una visión epidemiológica distinta, incorporando las categorías y etiologías de enfermedad desde el punto de vista de la población de diferente cultura.
\end{abstract}

Palabras clave: Salud de poblaciones indígenas, antropología, diversidad cultural, competencia cultural.

\begin{abstract}
This article tries to discuss and to reflect upon the way in which the immersed health system services in territories with high Mapuche presence are adapted to the cultural idiosyncrasy of this population, approaching which are the difficulties the services of health meet at the moment of exercising the health practice in multiethnic context, where the health is understood in an holistic way, leading necessarily to a different epidemiological vision, incorporating the categories and etiologies of disease from the the different population culture point of view.
\end{abstract}

Key words: Health of indigenous peoples, cultural anthropology, cultural diversity, cultural competence.

Fecha recepción: 17/03/12 Fecha aceptación: 26/12/12

\footnotetext{
${ }^{1}$ Este escrito nació de interrogantes sobre el tema, surgidas después de haber participado en un levantamiento de información, respecto a la prevalencia de trastornos de salud mental en las comunidades mapuches de la zona de la precordillera de la comuna de Panguipulli (XIV Región de los Ríos, Chile), solicitado por la Mesa de Salud Intercultural de Panguipulli en el año 2009.

•Antropólogo Social. Universidad Academia de Humanismo Cristiano. Email: nayip.hasen@gmail.com
} 


\section{INTRODUCCIÓN}

Comienzo por poner arriba de la mesa la imperiosa necesidad de la medicina occidental por asumir que los pueblos originarios tienen conceptos de salud-enfermedad diferentes, no individualistas, frente al cual el sistema de salud oficial muchas veces no cuenta con los códigos necesarios para entender su etiología, ni su asociación a múltiples factores espirituales, comunitarios y ambientales. Se hace necesario, por tanto, reconocer que ningún sistema médico es capaz de satisfacer por sí solo todas las demandas de salud que presenta una población, y por tanto el modelo oficial no es el único deseable y válido para comprender ciertos fenómenos.

Muchas veces identificamos, entre otras cosas, una enorme brecha de entendimiento entre la explicación que las propias comunidades hacen de sus enfermedades, y los diagnósticos, muchas veces errados, por parte de los agentes de los servicios de salud presentes en zonas de alta concentración indígena, entendiendo que en la cultura mapuche existe una noción mucho más holística respecto al tema, en la cual la enfermedad está más asociada a ámbitos comunitarios, espirituales y ambientales.

En esta oportunidad pretendo abordar la manera en la cual los servicios del sistema de salud inmersos en territorios con alta presencia de comunidades mapuche se adecuan a la idiosincrasia cultural de esta población, cuales son las dificultades con que se encuentran a la hora de ejercer la práctica de atención de salud en contextos pluriétnicos, y cuáles deberían ser (según la propia experiencia) los caminos a seguir para una adecuada epidemiología sociocultural, o lo que Ibacache (1) ha descrito como etnoepidemiología.

\section{EL CONCEPTO DE SALUD DESDE LA OMS}

Como bien lo plantea González de Rivera (2), la epidemiología como concepto se ha definido durante muchos años como una ciencia abocada únicamente a estudiar la naturaleza y la transmisibilidad de las enfermedades principalmente infecciosas dentro de los grupos humanos. Sin embargo, este mismo método se ha aplicado en la actualidad a la comprensión y el control de muchos otros trastornos, en particular aquellos en los que la relación del hombre con su ambiente interviene de manera significativa (2). En base a esto, el concepto de causalidad multifactorial de la patología humana ha sido plenamente asumido por el pensamiento epidemiológico, que entiende la salud y la enfermedad como dos resultados extremos de la compleja interacción del individuo en su entramado físico y psicosocial.

Por su parte, Jenicek (3) plantea que la epidemiología clásica y su objeto de estudio ha visto ampliado su campo de interés, hasta llegar a abordar problemas sanitarios derivados básicamente de factores socioculturales como la desigualdad, el estrés o la desadaptación de la persona frente a la sociedad, añadiéndose procedimientos apropiados no sólo para el estudio de las enfermedades crónicas, sino también para el extenso campo de la desadaptación, la vulnerabilidad y la calidad de vida (2).

Por su parte, el concepto de salud se ha definido de formas diversas de acuerdo a cada contexto cultural, concepto que abarca, entre otros aspectos, el bienestar subjetivo, la percepción de la propia eficacia, la autonomía, la competencia, la dependencia intergeneracional y la autorrealización de las capacidades intelectuales y emocionales (4). Esto dificulta el llegar a una definición exhaustiva 
de la salud desde una perspectiva transcultural. Sin embargo, se ha podido establecer que el concepto es mucho más amplio que la mera ausencia de trastorno o afección, conjugándose factores biológicos, psicológicos y sociales.

\section{INTERCULTURALIDAD Y CONCEPTO DE SALUD - ENFERMEDAD}

Como bien se establece en los lineamientos del Ministerio de Salud, integrar el enfoque intercultural a los programas de salud y salud mental, en especial en las zonas de alta concentración de pueblos originarios, resulta fundamental para la implementación de estrategias que consideren la visión de mundo de los usuarios. En este sentido, es necesario reconocer que las acciones de la atención primaria de salud se dan en contextos culturales diferenciados en constante interacción. El diseño de estrategias, programas y acciones definidos sólo en relación al sistema de creencias del equipo de salud, representa un obstáculo para el acceso, la oportunidad de la atención y más aún para la autogestión en salud de los usuarios pertenecientes a grupos étnicos minoritarios ${ }^{2}$.

Chile se constituye como un espacio pluriétnico, marcado por la coexistencia de distintos grupos étnicos caracterizados por visiones particulares de entender el mundo y actuar frente a él, situación que en el ámbito de la salud-enfermedad, se manifiesta en la coexistencia de diversas formas de abordar el tema. De esta manera, cuando estamos frente a una determinada cultura, nos encontramos frente a diversas creencias, conocimientos, percepciones y prácticas terapéuticas, validadas socialmente por quienes la sustentan (5).

\footnotetext{
${ }^{2}$ MINSAL. "Prevención de problemas y/o enfermedades de salud mental en adolescentes en el nivel primario de atención”. DIPRECE Depto. Salud Mental Depto. Ciclo Vital. Documento borrador; s/f.
}

Se entiende la interculturalidad como una relación entre culturas dinámicas, en la cual existe necesariamente reciprocidad, voluntad y horizontalidad, reconociendo que hay espacios de encuentro donde se pueden negociar y otros donde se mantienen las especificidades respetando las diferencias, mejorando la salud de ambos pueblos, ganándose espacios desde los pueblos originarios y cediendo espacio desde las instituciones oficiales (5).

El proceso de salud/enfermedad/atención es entendido como un hecho universal, que se desarrolla de forma particular y diferente en cada sociedad y en cada cultura. Todas las culturas han desarrollado, y desarrollan, modelos médicos a partir de los cuales entender y enfrentar la enfermedad y de ser posible recuperar la salud (6). En la cultura mapuche la salud es entendida de forma holística. Lo que se denomina Küme Mongen (o Buena Vida) es el resultado del equilibrio entre la persona, el medio ambiente y lo sobrenatural, integrando este enfoque en el modelo armonía-desarmonía, en donde la salud se mantiene como una interdependencia entre los elementos antes mencionados (7).

Ese equilibrio se permite a través de la estructuración de tres elementos: la salud, el desarrollo y la equidad (6). En este contexto, la salud se pierde cuando existe una transgresión o desequilibrio entre alguno de los tres elementos. Existe un comportamiento de protección que va mas allá del espacio y del tiempo, es decir que la protección se da tanto dentro del lof $^{3}$ como en su entorno (conceptualización particular del territorio y cada uno de los elementos contenidos en él).

Ibacache (7) plantea que el concepto de salud en la población mapuche trasciende el marco utilizado en la occidental, puesto que de lo que se trata es poder integrar tanto los

\footnotetext{
${ }^{3}$ El lof es la forma básica de organización social del pueblo mapuche. Se constituye como un grupo familiar (clan o linaje) que reconoce la autoridad de un lonco.
} 
elementos físicos y biológicos con el ámbito psicológico.

En este sentido, el diálogo entre la antropología sociocultural y la psiquiatría, por ejemplo, ha demostrado que los procesos de salud y enfermedad mental dependen del contexto sociocultural en el que se desarrollan, existiendo la necesidad de comprender y describir los procesos etiológicos y terapéuticos que sustenta la población mapuche en torno a los conceptos de salud y enfermedad, pudiéndolo contrastar con la explicación científica y occidental que caracteriza a la práctica de atención de salud moderna, comprendiendo y explicando sus convergencias y divergencias. Se trata, entonces, de que la práctica médica reconozca que los actores sociales perciben y conciben su falta de salud/enfermedad, desde una perspectiva emic, que puede ser radicalmente opuesta a las explicaciones científicas (8).

\section{DETERMINANTES SOCIALES E INTERCULTURALIDAD}

Es sabido que en América Latina las diferencias por clase social, etnia, género y edad han dado origen a desigualdad y exclusión. Chile no es excepción, aun cuando se ha avanzado relativamente en abandonar la invisibilidad del problema que se expresa en la inequidad que traen aparejadas esas desigualdades. La tarea es reconocer dónde se producen las brechas para incorporar procesos de integración social. Una de ellas son las brechas en salud expresadas en inequidad en el acceso a servicios y exclusión por razones culturales, muchas veces producto de desconocimiento o subestimación de la cosmovisión indígena de la salud (9).

Se producen barreras en la comunicación y calidad de trato, por códigos culturales distintos, rituales, símbolos, rol de las familias y comunidades, percepciones sobre el cuerpo y el pudor de las mujeres indígenas mu- chas veces incomprendidos, o por estilos de crianza distintos. Frente a este panorama, se requiere un reconocimiento y valoración de los conocimientos y saberes de sistemas de salud tanto occidental como indígena, siempre apuntando a una complementariedad de ambos mundos (10).

Es indispensable, por tanto, que el profesional de la salud esté informado acerca de los derechos que el Estado, sus instituciones centrales y convenciones internacionales, les otorgan a estas personas, y las razones que fundamentan tales derechos (11).

Chile cuenta, desde salud, con normativas específicas como la Norma General Administrativa $\mathrm{N}^{\circ} 16$ sobre interculturalidad en los Servicios de Salud (12), el cual en uno de sus artículos señala: "En la formulación de los Planes de Salud Pública regionales, la Autoridad Sanitaria Regional velará por la incorporación de estrategias y actividades destinadas a identificar, monitorear y en lo posible a disminuir las brechas de equidad en la situación de salud y medio ambiente de la población indígena, y proponer las medidas intersectoriales pertinentes" (12).

\section{INTERCULTURALIDAD EN SALUD: DIFICULTADES Y FALENCIAS}

Derivado del reconocimiento y el llamado realizado por la $\mathrm{OMS}^{4}$ de avanzar en la generación de estrategias nacionales en materia de salud de los pueblos indígenas, en conjunto con las iniciativas adoptadas por el Servicio de Salud Araucanía en el Programa con Población Mapuche y los mandatos de

\footnotetext{
${ }^{4}$ A partir de 1993, año en el cual se aprobó la iniciativa de Salud de los Pueblos Indígenas, la OMS (Organización Mundial de la Salud) y los estados miembros se comprometieron a trabajar con la población indígena, con el objetivo de mejorar su salud y bienestar, reconociendo el valor del patrimonio cultural y el saber ancestral de los pueblos indígenas y la necesidad de conservarlos. Ministerio de Salud Chile; 2006 .
} 
la Ley Indígena (13), es que a partir del año 1996 comienza a implementarse una línea de trabajo por parte del Ministerio de Salud, el cual impulsa un Programa Especial de Salud y Pueblos Indígenas, dedicado a la generación de orientaciones técnicas y políticas en salud intercultural a nivel nacional, el cual cuenta con la participación de 22 Servicios de Salud, en cuyas áreas geográficas se encuentra la mayor concentración de población indígena (14).

Sin embargo, como plantea Citarella (15), ¿es posible adecuar los servicios del sistema de salud oficial a la idiosincrasia cultural de la población indígena y a la vez compatibilizar la cultura mapuche con la cultura nacional occidental? En este sentido, ¿qué significaría la interculturalidad en salud o salud intercultural?

La interculturalidad en salud requiere del desarrollo de procesos donde los propios pueblos originarios y los sistemas médicos oficiales expresan la voluntad de encontrarse en una relación horizontal. Un principio rector en que se puede manifestar la interculturalidad en salud es la complementariedad de estos sistemas médicos, proponiendo un acercamiento de los equipos de salud (medicina occidental) con los especialistas y terapeutas de la medicina tradicional, respetando los conocimientos del otro y generando espacios de confianza que den lugar a la complementariedad entre ambas medicinas (14).

Así, la formación de recursos humanos en salud con orientación intercultural supone mejoras en la competencia técnica y humana del personal institucional, incidiendo en el respeto, en el trato de los usuarios, en el reconocimiento de las tradiciones culturales, en el combate a la exclusión, en la atención y la equidad en salud a los distintos grupos étnicos (16).

La capacitación del personal, para que produzca un impacto positivo, deberá incidir en cambios importantes en la prestación de los servicios, mejorando la relación médi- co-paciente y paciente-institución de salud, es decir, brindar atención con sensibilidad intercultural (17).

Dentro de este marco, la figura del facilitador intercultural ${ }^{5}$ se torna trascendental, siendo el nexo entre el equipo de salud y las comunidades indígenas, a la vez que pertenece a las comunidades y es avalada por estas mismas. Lo cual en términos operacionales, como lo plantea Álvarez (18), implica potenciar lo común entre el sistema médico occidental y el sistema médico indígena (colocar referencia), respetando y manteniendo la diversidad.

Ibacache (1) esboza que para el conocimiento del impacto que puede tener sobre las personas el desequilibrio entre factores protectores y transgresores en un contexto intercultural, se proyecta el desarrollo de una visión epidemiológica distinta, con una teoría de la causalidad de la enfermedad más amplia, comprensiva e integral, reconociendo la historia y maneras de entender la salud-enfermedad, junto a sus determinantes socioculturales. De esta forma se propone una Etno-epidemiología o Epidemiología Intercultural, incorporando las propias categorías y etiologías de enfermedad desde el punto de vista de la población de diferente cultura.

Es necesario que los agentes que se desempeñan en la atención en salud en zonas de alta concentración de población indígena logren una mayor comprensión de la diversidad y dinámica cultural y social en la que están insertos los usuarios de salud. Es en este marco en el que se han implementado procesos de formación y capacitación de los funcionarios de salud hacia lo que es la salud intercultural, tendientes a favorecer las

\footnotetext{
${ }^{5}$ En el año 1996, con la creación del Programa de Salud y Pueblos Indígenas los facilitadores interculturales comienzan a institucionalizarse, hecho que se acrecienta aún más con la promesa presidencial, a fines del año 1999, de insertar facilitadores interculturales en todos los establecimientos de salud ubicados en territorios con población indígena significativa.
} 
estrategias de comunicación entre cuerpo de salud oficial y pueblos indígenas, logrando entre otras cosas una mejora en la satisfacción usuaria de la población atendida, mayor calidad en el trato y disminución del prejuicio étnico.

\section{CONSIDERACIONES FINALES}

En nuestro país, la mejora de la salud mediante una gestión más adecuada del medio ambiente local es un camino raramente explorado por los organismos a cargo de los programas de salud, existiendo una escasa identificación de la red de factores económicos, sociales y ambientales que influyen en la salud humana, presentándose dificultades a la hora de identificar estos temas como factores influyentes en la salud, debido a que muchas veces las poblaciones indígenas no manifiestan de manera clara, frente a los agentes de salud oficial, las causas que originan sus estados de salud, y a una incapacidad de los agentes oficiales de la salud de considerar estas causas como gatillantes a la hora de realizar un diagnóstico acertado.

El diagnóstico de necesidades tendría que constituir el punto de partida para cualquier intervención comunitaria en salud. Sin embargo, existen omisiones hechas a nivel de salud que nos sugieren algunas reflexiones críticas respecto al tema.

Por un lado se debe asegurar la implementación y fortalecimiento de acciones al interior de las propias comunidades, con trabajo en las sedes sociales y establecimientos educacionales, con la finalidad de prevenir problemas de salud, a través de la capacitación y sensibilización tanto de las comunidades como de los agentes de salud oficial en temas de salud intercultural.

Sin embargo, no basta sólo con la sensibilización de los profesionales en temas de salud intercultural, también se hace necesario una real capacitación en un contexto in- terdisciplinario, que capacite realmente a los agentes de los servicios públicos a desenvolverse en contextos comunitarios e interculturales, permitiendo un mejor diagnóstico de ciertas enfermedades, teniendo en cuenta los procesos etiológicos y terapéuticos que sustenta la población mapuche en torno a los conceptos de salud y enfermedad.

Se hace necesario, además, promover aún más, a nivel de institución y organismos de la salud, las investigaciones orientadas a entender las relaciones entre los ecosistemas, sus usos y funciones por parte de las poblaciones, y su relación directa con los temas de salud (física y mental).

Para esto debe considerarse toda la información pertinente, incluidos los conocimientos, las innovaciones y las prácticas de las comunidades científicas, indígenas y locales en relación a los aspectos promocionales, preventivos, curativos y de rehabilitación, desde un enfoque de salud ecosistémica (19).

Metodológicamente hablando, se propone una estrategia orientada a la investigación-acción participativa, es decir, la acción conjunta del investigador, o los profesionales de la salud, con los informantes, en las distintas etapas del proceso de investigación, lo cual abarca la familiarización de ambas partes, la formulación de los temas que se desean abordar (demandas sentidas de la población), la forma de hacerlo, las acciones orientadas en tal sentido, su evaluación y la generación de conocimientos derivado de las experiencias (20). Esto incluye la reflexión de todos los actores en torno a sus problemas, necesidades y recursos, haciendo una reivindicación del saber popular o conocimiento que manejan acerca de la realidad, sacando a los profesionales de los servicios públicos de la relación verticalista médico-paciente, llevándolos a un real trabajo en terreno y de acercamiento a las comunidades, entendiendo que la enfermedad no se cura una vez los pacientes salen del box de atención, sin hacer un seguimiento real y una identificación eficaz de los factores relevantes en contexto. 
En resumen, este cambio de relación de "investigador" a "facilitador" conlleva tres aspectos claves: el uso de métodos participativos apropiados, un cambio de actitudes y el intercambio de información entre todos los actores.

\section{REFERENCIAS}

1. Ibacache J. Rume kgen mew ta az mapu/ Epidemiología de la transgresión en Maquehue-Pelale [Internet]. Temuco, Chile: Unidad de salud con Pueblos indígenas. Servicio de Salud Araucanía Sur; 2001. 21 p. [citado 2 febrero 2012]. Disponible en: http://www.fcs.uner.edu.ar/revistamaestriasfc

2. González de Rivera JL, Rodríguez Pulido F, Sierra López A. El método epidemiológico en Salud Mental. Barcelona: Editorial Masson-Salvat; 1993. 444 p.

3. Jenicek M, Cléroux R. Epidemiología: Principios, técnicas y aplicaciones. Barcelona: Salvat Editores; 1987. 393 p.

4. Organización Mundial de la Salud. Informe sobre la Salud en el Mundo 2001. Salud Mental: nuevos conocimientos, nuevas esperanzas [Internet]. Ginebra: OMS; 2001. 178 p. [citado 2 febrero 2012]. Disponible: http://www.who.int/whr/2001/ es/index.html

5. Davinson G, Jélvez I, Yánez S. Diagnóstico y propuesta de sistema de salud intercultural para Indígenas. Bases para una propuesta de un Seguro Indígena. Temuco: Instituto de Estudios Indígenas; 1997. $145 \mathrm{p}$.

6. Cetti A. La salud intercultural mapuche a través del tamiz de la gubernamentalidad. Un estudio de caso [Internet]. Ponencia presentada en: 3ras Jornadas de Historia de la Patagonia; 6-8 noviembre 2008; Bariloche, Argentina. [citado 5 febrero 2012]. Disponible: http://www.hechohistorico.com.ar/.
7. Ibacache J, Morros Martel L, Trangol Namuncura M. Salud Mental y enfoque socioespiritual-psico-biológico. Una aproximación ecológica al fenómeno de la salud-enfermedad desde los propios comuneros y especialistas terapéuticos mapuches de salud [Internet]. Nuke Mapuforlaget Working Paper Series 11; 2002 [citado 26 febrero 2012]. Disponible en: http://www.mapuche.info/mapuint/ sssmap020911.pdf. 91-89629-12-4

8. García Vázquez C, Saal A. Transculturalidad y enfermedad mental: los mapuches de Neuquén. Revista de la Facultad. 2007; 13: 35-37.

9. Ministerio de Salud. Antecedentes para apoyar la revisión de los programas de salud pública desde la perspectiva de equidad y determinantes sociales. Chile: Subsecretaría de Salud Pública; 2009.

10. Rodríguez L. Enfoque Intercultural en Políticas de Salud Materna [Internet]. Seminario-taller Pueblos indígenas de América Latina: Políticas y programas de salud ¿cuánto y cómo se ha avanzado?; 25-26 junio 2007; Santiago, Chile: CELADE - FI [citado 4 febrero 2012]. Disponible en: http: //www.eclac.cl/celade.

11. Durán T. Pertinencia cultural en las Políticas de Infancia en Chile. Temuco: Escuela de Antropología, Universidad Católica de Temuco; s/f.

12. Ministerio de Salud. Norma General Administrativa $\mathrm{N}^{\circ} 16$. Interculturalidad en los Servicios de Salud. Resolución Exenta $\mathrm{N}^{\circ} 261$ [citado 5 marzo 2012]. Disponible en: www.minsal.cl/juridico/RESOLUCION_261_06.doc

13. Ley No 19.253/1993 Establece Normas sobre Protección, Fomento y Desarrollo de los Indígenas, y crea la Corporación Nacional de Desarrollo Indígena. Biblioteca Congreso Nacional [citado 15 julio 2011]. Disponible en: http://www.bcn.cl/

14. Ministerio de Salud. Políticas de Salud y Pueblos Indígenas. Santiago: Gobierno de Chile - Ministerio de Salud; 2003. 
15. Citarella L, coord. Medicinas y cultura en la Araucanía. Santiago, Chile: Editorial Sudamericana; 1995.

16. Lerin Piñon, S. Desafíos de la salud intercultural [Internet] $14 \mathrm{p}$. [citado 10 marzo 2012]. Disponible en: http://www.geocities.ws/congresoprograma/8-1.pdf

17. Lerin Piñon S. Interculturalidad y Salud. Recursos adecuados para la población indígena o propuestas orientadas a opacar la desigualdad social [Internet]. $11 \mathrm{p}$. [citado 10 marzo 2012]. Disponible en: http://www.ops.org.bo/multimedia

18. Álvarez Díaz N. Interculturalidad en Sa- lud y Desarrollo de Comunidades Mapuche: Estudio de Caso en Makewe Pelale. [Tesis Magíster]. [Temuco]: U. Católica de Temuco; 2005. 109 p.

19. Lebel J. Health: an ecosystem approach. Canadá: International Development Research Centre. 2003.84 p.

20. Hasen F. Sistematización Reunión Nacional del Consejo Consultivo de Adolescentes y Jóvenes [Internet]. Santiago: Ministerio de Salud, Chile; 2011. 104 p. [citado 2 febrero 2012]. Disponible en: http://www.minsal.gob.cl 